\title{
Cervical Decompression for Tandem Spinal Stenosis: The Impact on Low Back Pain
}

Matthew D. Alvin, MBA, MS, MA ${ }^{1,2}$; Vincent J. Alentado, BS ${ }^{1,2}$; Daniel Lubelski, BA ${ }^{2,3}$; Edward C. Benzel, MD²,3, Thomas E. Mroz MD',3,4*

${ }^{1}$ Case Western Reserve University School of Medicine, Cleveland, Ohio, USA

${ }^{2}$ Cleveland Clinic Center for Spine Health, Cleveland Clinic, Cleveland, Ohio, USA

${ }^{3}$ Cleveland Clinic Lerner College of Medicine, Cleveland, Ohio, USA

${ }^{4}$ Department of Neurological Surgery, Cleveland Clinic, Cleveland, Ohio, USA

*Corresponding Author: Thomas E. Mroz, MD

Neurological Institute

Cleveland Clinic Center for Spine Health

Departments of Orthopaedic and Neurological Surgery

The Cleveland Clinic

9500 Euclid Avenue, S-80

Cleveland, Ohio 44195

Tel: $216-445-9232$

Fax: 216-363-2040

Email: mrozt@ccf.org

IRB Approval \#12-1240. No grants, technical or corporate support were received in conducting this study or writing this manuscript. No conflicts of interest existed relative to the present study. 
$\underline{\text { Abstract }}$

Study Design. Retrospective analysis.

Objective. To determine the impact of cervical spine surgery on both neck and low back pain in patients with symptomatic tandem spinal stenosis

Summary of Background Data. Tandem spinal stenosis (TSS) can present similarly to cervical spondylotic myelopathy, but often has a worse prognosis. Few studies have investigated outcomes and compared treatment approaches for patients with TSS.

Methods. Eighty-four patients with clinical and imaging evidence of TSS were identified between 2008 and 2013. Of those identified, 48 underwent cervical spine surgery alone, 20 underwent both cervical and lumbar spine surgery, and 16 received conservative treatment alone (conservative cohort). Quality of life (QOL) scores including the Visual Analogue Scale (VAS) for arm, neck, and back, Pain Disability Questionnaire (PDQ), Patient Health Questionnaire-9 (PHQ-9), and EuroQOL-5 Dimensions (EQ-5D) were acquired via an institutional prospectively collected database.

Results. Average follow-up for all three cohorts was twelve months. The average duration of symptoms prior to treatment for all patients was 17 months (range: 1-120 months). 90\% of patients underwent cervical fusion and $88 \%$ received multi-level operations. All patients presented with myelopathic symptoms. Both surgical cohorts showed significant $(\mathrm{p}<0.01)$ pre- to postoperative improvement for VAS neck and arm scores at 1-year post-op and significantly greater improvements than the conservative cohort. In addition, the cohort undergoing cervical spine surgery alone experienced significant improvement in the EQ-5D score whereas those undergoing both cervical and lumbar spine surgery did not. Low back pain stayed the same or worsened for both surgical cohorts at both the initial postoperative visit as well as through the final follow-up.

Conclusions. Cervical spine surgery with or without follow-up lumbar spine surgery significantly improves neck pain in patients with TSS. In contrast, cervical spine surgery in these patients does not improve low back pain. Rather, it may unmask lumbar symptoms leading to subsequent lumbar spine surgery. In our cohort of TSS patients, lumbar surgery also did not improve low back pain or quality of life. Future prospective studies are necessary to confirm these findings and examine the impact of lumbar decompression alone on cervical spine symptoms in patients with TSS.

Key words. tandem spinal stenosis, quality of life, outcomes, spinal stenosis, fusion 


\section{$\underline{\text { Introduction }}$}

Symptomatic tandem spinal stenosis (TSS) is a clinical entity in which both the cervical and lumbar portion of the spinal canal is narrowed. TSS occurs in $5-28 \%{ }^{2}$ of all people. Patients usually present with a triad of neurologic signs of both upper and lower motor neuron dysfunction, neurogenic claudication, and progressive gait disturbances. Commonly, either cervical (myelopathy) or lumbar (claudication) symptoms initially predominate. When these initial symptoms are treated surgically, complaints related to the secondary site of stenosis arise. Patients undergoing treatment for TSS may undergo staged (cervical prior to lumbar or vice versa) or simultaneous surgery.

Previous studies have shown similar positive outcomes for staged and simultaneous surgical approaches for treatment of patients with TSS. ${ }^{3-5}$ When using a staged approach, the initial decompression is the more symptomatic site. Alternatively, if both sites are equally symptomatic, the cervical spine may be decompressed first, as there are patient reports of postoperative reduction of the lumbar symptoms in addition to the cervical symptoms. ${ }^{6}$

Studies have shown that cervical decompression with or without fusion can improve symptoms of neck pain or radiculopathy in patients with either cervical stenosis alone or TSS. ${ }^{3-5}$ However, the effects of cervical decompression on lower back pain in patients with TSS have only been minimally investigated. Moreover, there have been no controlled studies investigating these proposed effects. Accordingly, in the present study, we hypothesized that cervical decompression surgery can lead to improvement of both neck and lower back pain as well as overall quality of life.

\section{Methods}

Electronic medical records were retrospectively reviewed to identify patients with TSS using both current procedural terminology (CPT) and international classification of diseases (ICD-9) codes for patients with radiculopathy or neck pain undergoing cervical spine decompression with or without fusion. Once identified, demographic and clinical information on each patient was collected. Diagnosis of tandem spinal stenosis was made both clinically and radiographically via magnetic resonance imaging (MRI). Patients needed to have both clinical and imaging evidence to be included.

Patients were divided into three cohorts: those that underwent cervical spine surgery, those that underwent cervical spine surgery and subsequent lumbar spine surgery, and those that did not undergo surgery (conservative cohort). Patients were excluded if they were younger than 18 years or older than 80 years, had previous spine surgery, non-spondylotic causes of radicular pain (e.g., tumor, infection), neuromuscular disease (e.g., multiple sclerosis), or a workers' compensation claim.

Quality of life (QOL) scores including the Visual Analogue Scale (VAS) for arm, neck, and back, Pain Disability Questionnaire (PDQ), Patient Health Questionnaire-9 (PHQ-9), and EuroQOL-5 Dimensions (EQ-5D) were acquired via the institutional Knowledge Program (KP). The KP is a patient derived outcome assessment tool that is embedded in our electronic medical 
record. For all measures, except the EQ-5D, a decrease in score represents improvement. These data have been systematically collected since 2009 , in a prospective fashion, at the time of the patient visits. The minimum clinically important difference (MCID) used for each questionnaire in a 1-year time frame was as follows: VAS (2.6), PDQ (26), PHQ-9 (5), and EQ-5D (0.1). ${ }^{7-9}$ Demographic variables (categorical data) between cohorts were compared using Fisher's exact tests. Quality of life outcomes (continuous data) between cohorts were compared using the Student's t-test and one-way ANOVA analyses with post hoc paired t-tests and Tukey simulations. All p-values $\leq 0.01$ were considered statistically significant to adjust for multiple comparisons.

\section{$\underline{\text { Results }}$}

\section{Demographics}

Eighty-four patients with clinical and MRI evidence of TSS were identified. Included patients exhibited signs and symptoms of both cervical and lumbar stenosis. Sixty-eight underwent surgery while 16 did not (Table 1). The average age of patients for the three cohorts was 61.2, 63.9 , and 53.8, respectively $(\mathrm{p}=0.01)$, with a significantly greater percentage of males in the surgical cohorts $(64.6 \%$ and $65 \%$ vs. $25 \%$; $=0.01)$. The average duration of symptoms (myelopathy and low back pain) prior to the date of hospital presentation was 17 months and ranged from 1 month to 120 months for all three cohorts. In the surgical cohorts combined, 90\% of patients underwent cervical fusion and $88 \%$ received multi-level operations (average 2.6 levels). The number of levels operated did not impact outcomes. No patient received simultaneous cervical and lumbar operations. Six neurosurgeons and five orthopaedic surgeons, all fellowship trained in spine surgery, performed all operations (with equal distributions of the surgical approaches and levels). After undergoing cervical fusion (90\%) or cervical decompression only (10\%), 20 patients $(29 \%)$ subsequently underwent lumbar surgery. The average follow-up for the three cohorts (cervical, cervical/lumbar, conservative) was 10.9, 11.6, and 15.1 months, respectively.

\section{Health-related Outcomes}

The average pre- to postoperative change in scores for each questionnaire (cervical, cervical/lumbar, conservative cohorts, respectively) were: VAS Arm (-3.3, -4.4, 0.50), VAS Neck (-3.1, -4.4, 0.19), VAS Low Back (0.02, -1.2, -0.27), PDQ (-8.8, -5.7, -0.85), PHQ-9 (-1.2, $1.1,1.9)$, and EQ-5D $(0.13,0.03,0.02)$. At 1-year post-op, both surgical cohorts had statistically significant $(\mathrm{p}<0.01)$ and clinically significant (Change score $>$ MCID) improvement in VAS arm and neck scores while the conservative cohort had no improvement. In addition, the cohort undergoing cervical surgery alone had statistically and clinically significant improvement in the EQ-5D. Low back pain VAS scores did not improve after cervical surgery alone, but did improve non-significantly after lumbar surgery. On the contrary, cervical spine surgery was associated with worsening low back pain VAS scores. Lumbar spine surgery performed after cervical spine surgery did not lead to greater improvement in quality of life in any outcome measure other than the low back pain VAS score as compared with cervical spine surgery alone. For the low back pain VAS score, the improvement after both cervical and lumbar surgery did not surpass the cohort's baseline low back pain VAS score. 


\section{$\underline{\text { Discussion }}$}

Epstein et al. ${ }^{10}$ reported outcomes of 20 patients with TSS who underwent cervical decompression, and found that 12 patients $(60 \%)$ experienced improvement of lower extremity symptoms, as well as relief of spasticity and myelopathy. No QOL outcomes or statistical analysis was performed. The authors suggested that the improvement in the lower extremity symptoms might have been due to relief of the posterior column and corticospinal tracts in the cervical spine. At two-year follow-up, none of the patients required lumbar decompression. However, the other eight patients, who experienced initial improvement for 6-9 months postoperatively, developed significant lumbar complaints impairing ambulation and required subsequent lumbar laminectomy. The patients that required a second surgery were an average of 10 years older than the rest of the cohort. In the present study, patients requiring subsequent lumbar surgery were no different in age than those requiring only cervical surgery. In addition, lumbar symptoms worsened rather than improved after the initial cervical surgery.

Dagi et al. ${ }^{11}$ retrospectively reviewed 19 patients who underwent staged surgery for TSS and had 22-month follow-up. Nine patients (50\%) reported subjective improvement, five $(25 \%)$ worsened, and five (25\%) remained unchanged (no QOL outcome measures or p-values reported). Improvement correlated inversely with symptom duration. Three patients that underwent cervical decompression initially did not require subsequent lumbar decompression while the other sixteen patients required both cervical and lumbar surgery. In the present study, we used multiple objective QOL outcome measures and found no relationship between symptom duration and improvement. Those requiring subsequent lumbar surgery had no improvement in overall quality of life (EQ-5D) after the initial cervical surgery while those that only underwent cervical surgery experienced significant improvement in overall quality of life.

There have been few additional studies investigating TSS the literature. LaBan and Green ${ }^{2}$ performed a 10-year review of 460,964 hospital admissions and found only 54 patients (36 men, 18 women) were diagnosed with MRI-confirmed TSS $(0.12 \%)$, which were identified via ICD diagnosis codes. Fifty-one patients (94\%) were over the age of 51, indicating the disease is more prevalent in the older age group. The authors did not review surgical outcomes. Similarly, our patient cohort was comprised of an older and male-dominated group of patients. Other case reports have been performed that show improvement after either staged or simultaneous surgery. To compare simultaneous versus staged surgery, Eskander et al. ${ }^{3}$ evaluated 43 patients with TSS (21 simultaneous; 22 staged) over a mean follow-up of seven years and found significant improvements in Japanese Orthopedic Association (JOA) and Oswestry Disability Index (ODI) scores for both cohorts. No significant differences existed between cohorts for JOA or ODI scores, as well as frequency of major or minor complications.

While TSS is a relatively rare clinical entity, it is currently unclear how to best treat patients with concurrent cervical and lumbar symptoms. Krishnan et al. ${ }^{14}$ retrospectively analyzed 53 patients who underwent single-stage simultaneous surgery for TSS. Quality of life outcomes recorded with 36-month follow-up included modified JOA (mJOA), ODI, patient satisfaction index, and Nurick's grade. Statistically significant postoperative improvement was seen for all measures $(\mathrm{p}<0.01)$. Patients older than 60 were more likely to experience complications. In the present 
study, we used different QOL outcome measures with approximately 12 month follow-up and found significant improvement in quality of life after cervical surgery alone but not after lumbar surgery, indicating that subsequent lumbar surgery is likely unnecessary for improvement in quality of life in patients with TSS.

Rather than improving after cervical surgery as expected given prior study findings, low back pain stayed the same or even trended to a worsening state for some patients. There may be a physiological manner in which decompressing the cervical spine could relieve impingement on descending pathways that cause pain in the lumbar region. ${ }^{6}$ However, it is also possible that decreasing one source of pain (cervical) may unmask a second source (lumbar) that was initially unrecognized. Patients requiring subsequent lumbar surgery had lower improvement in QOL and reported worsened low back pain compared to the visit prior to cervical spine surgery. However, these patients had the same improvement in neck pain as did the cohort that underwent cervical surgery alone.

We acknowledge certain limitations in the present study. First, the retrospective nature of the design opens the study to bias and limited inclusion only to those patients with complete data sets. Second, our patient population varied relative to surgeon, type of surgery, age, and followup time. However, all of these factors were statistically analyzed separately and found to not impact the conclusions reached in our study. Despite these limitations, this study includes the largest sample size of patients with TSS to date, is the first controlled study to compare surgery with conservative management, and is the first study to show that subsequent lumbar surgery provides no benefit to patients with TSS who underwent cervical surgery.

\section{$\underline{\text { Conclusion }}$}

Cervical spine surgery with or without follow-up lumbar spine surgery significantly improves neck pain in patients with TSS. Cervical spine surgery in these patients, however, does not improve their low back pain. On the contrary, it may unmask lumbar symptoms leading to subsequent lumbar spine surgery. In our cohort of TSS patients, lumbar spine surgery did not improve low back pain or quality of life. Future prospective studies are necessary to validate these findings and examine the impact of lumbar decompression alone on cervical spine symptoms in patients with TSS.

\section{$\underline{\text { References }}$}

1. Teng P and Papatheodorou C. Combined cervical and lumbar spondylosis. Arch Neurol 1964;10:298-308.

2. LaBan MM and Green ML. Concurrent (tandem) cervical and lumbar spinal stenosis: a 10-yr review of 54 hospitalized patients. Am J Phys Med Rehabil 2004;83:187-90.

3. Eskander MS, Aubin ME, Balsis SM, et al. Is there a difference between simultaneous or staged decompression for combined cervical and lumbar stenosis. J Spinal Disord Tech 2011;24:409-13.

4. Hsieh $\mathrm{CH}$, Huang TJ, Hsu RW. Tandem spinal stenosis: clinical diagnosis and surgical treatment. Changgeng Yi Xue Za Zhi 1998;21:429-35. 
5. Kikuike K, Miyamoto K, Hosoe H, Shimizu K. One-staged combined cervical and lumbar decompression for patients with tandem spinal stenosis on cervical and lumbar spine: analyses of clinical outcomes with minimum 3 years follow-up. J Spinal Disord Tech 2009;22:593-601.

6. Langfitt TW and Elliott FA. Pain in the back and legs caused by cervical spinal cord compression. JAMA 1967;200:382-5.

7. Parker SL, McGirt MJ. Determination of the minimum improvement in pain, disability, and health state associated with cost-effectiveness: introduction of the concept of minimum cost-effective difference. Neurosurgery 2012; 71:1149-55.

8. Parker SL, Godil S, Shau DN, Mendenhall SK, McGirt MJ. Assessment of the minimum clinically important difference in pain, disability, and quality of life after anterior cervical discectomy and fusion. J Neurosurg Spine 2013; 18:154-160.

9. Wilson HD. Minimum clinical important differences of health outcomes in a chronic pain population: Are they predictive of poor outcomes? UT Arlington Dissertation 2008.

10. Epstein WE, Epstein JA, Carras R, et al. Coexisting lumbar and cervical spinal stenosis" diagnosis and management. Neurosurgery 1984;15:489-96.

11. Dagi TF, Trakington MA, Leech JJ. Tandem lumbar and cervical spinal stenosis: natural history, prognostic indices, and results after surgical decompression. J Neurosurg 1987;66:842-9.

12. Naderi S and Mertol T. Simultaneous cervical and lumbar surgery for combined symptomatic cervical and lumbar spinal stenosis. J Spinal Disord Tech 2002;15:229-32.

13. Aydogan M, Ozturk C, Mirzanli C, et al. Treatment approach in tandem (concurrent) cervical and lumbar spinal stenosis. Acta Orthop Belg 2007;73:234-7.

14. Krishnan A, Dave BR, Kambar AK, Ram H. Coexisting lumbar and cervical stenosis (tandem spinal stenosis): an infrequent presentation. Retrospective analysis of singlestage surgery (53 cases). Eur Spine J 2014;23:64-73.

15. Krieg SM and Meyer B. Quest for level I evidence in the treatment of cervical spondylotic myelopathy. World Neurosurg. 2014;81:501-2.

16. Alvin MD, Lubelski D, Benzel EC, Mroz TE. Ventral fusion versus dorsal fusion: determining the optimal treatment for cervical spondylotic myelopathy. Neurosurg Focus. 2013;35:E5. 